\title{
The role of genetic counseling in the early detection of fetal malformations
}

\author{
Gladys C. AL JASHI ${ }^{1}$, Isam AL JASHI ${ }^{2,3}$, Andra BORDEIANU ${ }^{2}$ \\ 1"Sf. Pantelimon" Emergency Clinical Hospital Bucharest, Romania \\ ${ }^{2}$ Faculty of Medicine, "Titu Maiorescu" University, Bucharest, Romania \\ ${ }^{3}$ Regina Maria Private Health Network, Bucharest, Romania
}

\begin{abstract}
Introduction. Genetic counseling is a communication process developed by specialists in the field, a process that laid the foundation for human and psychological issues arising from the presence of genetic malformations or the risk of such a disease in the family. Genetic counseling is a complex and specialized medical act. Most of the time, genetic counseling is given by one or more specialized people, the main purpose being to help the individual or the family (1).

The objectives of the study are to highlight the importance of knowledge of prenatal risks and possible pathologies by future parents, especially if they have knowledge of the existence of hereditary diseases in the family. The main objective is to analyze the risk factors and to establish whether or not to stop the tasks.

Materials and methods. We carried out a retrospective descriptive observational study, we analyzed all observation sheets of the patients who had an amniocentesis, which were recorded by the Obstetrics and Gynecology Department of the Ilfov County Hospital in Bucharest, Romania for a period of four years, namely January 2012-December 2015.

Results. In the four analyzed years, a total of 6,097 pregnancies were recorded, of which 408 had an indication of an amniocentesis in order to establish a correct diagnosis. The dynamics of the number of tasks during the analyzed period show significant decreases compared to the first year, with $-14.65 \%$ in 2014 and $11.15 \%$ in 2015.

Conclusions. According to the literature, screening tests have shown a margin of error in the diagnosis of fetal malformations, so the diagnosis of certainty results from amniocentesis. Most specialists consider that amniocentesis is an invasive method, so use of this diagnostic method should be reduced. Therefore, amniocentesis is recommended as an invasive prenatal diagnosis when the risk of a detectable abnormality by this method is considered greater than the procedural risk of amniocentesis. The final results of the 408 cases, about the post-amniocentesis pregnancy to pregnancy endpoint, claim that genetic amniocentesis presents a minimal risk of abortion or maternal infections, and fetal lesions are very rare.
\end{abstract}

Keywords: amniocentesis, fetal malformations, screening tests

\section{INTRODUCTION}

Prenatal pathology is represented by congenital illness, hereditary disease, and family illness. Congenital diseases or malformations are present at birth (they are not synonymous with heredity) and are the consequence of genetic or environmental factors. Not all genetic diseases are con- genital, especially with regard to the pathological phenotypic phenomenon at birth.

Hereditary disease is synonymous with transmitted or inherited disease and is the consequence of a transmitted genetic mutation. Some chromosomal abnormalities may also be inherited (translocation 15/21 leads to the occurrence of hereditary and familial cases of Down syndrome). 
Family illness is not synonymous with heredity or genetics because it can also be produced by factors of another nature, such as the action of environmental factors, identical to several members of the same family, common hirsutism, hippocampus.

Genetic counseling is a communication process developed by specialists in the field, a process that laid the foundation for human and psychological issues arising from the presence of genetic malformations or the risk of such a disease in the family. Genetic counseling is a complex and specialized medical act. Most of the time, genetic counseling is given by one or more specialized people, the main purpose being to help the individual or the family (1).

By giving the genetic counseling, the specialist physician first attempts to make future parents understand the nature of the condition they face or will face, and what is the probable evolution of it, but not least, the possibilities of care for the affection (2). Another priority objective of the genetic counseling is to explain the couple so that they understand the genetic factors that led to the affection, the mechanisms by which the disease is transmitted, and also the risk of the affection to other members of the family. One of the ultimate goals of this medical act is to correct as well as possible the progression of the affliction to the person affected, or to impose the necessary measures to limit as much as possible the risk of recurrence of the disease and to other members of the family (1).

Approximately half of these anomalies are detected at birth, while the rest become apparent later in childhood or rarely in adulthood. In families at risk, for a genetic anomaly, the probability of having an affected child may more than exceed the overall risk in the population, and therefore, in these families, procedures for prenatal diagnosis should be strictly applied (4).

The purposes of prenatal examinations are diagnostic, therapeutic, and genetic modification, and due to the fact that the affected individual ultimately pays tribute to genetic disability or embryo-fetal harm for the rest of his life, their importance is distinctive.

Progressions in prenatal diagnosis have been based on developing and refining less invasive diagnostic methods such as ultrasound, serum screening of aneuploidy, and Doppler assessment of the average cerebral artery to exclude fetal anemia. However, despite these safer techniques, amniocentesis remains an essential tool in prenatal assessment and diagnosis.

\section{OBJECTIVES OF THE STUDY}

Highlight the prevention and treatment of fetal malformations as early as possible in order to reduce their incidence so that each family can enjoy healthy children. Another objective is to emphasize the importance of knowing prenatal risks and possible pathologies by future parents, and analyzing the family, physiological, and pathological history of patients, which may be relevant in establishing important conclusions.

\section{MATERIALS AND METHODS}

In order to accomplish this descriptive observational study, we analyzed all observation records with an amniocentesis (an invasive procedure to accurately diagnose the diagnosis of a genetic abnormality) found in the Department of Obstetrics and Gynecology at the Ilfov County Hospital, on a period of four years, namely January 2012 - December 2015.

The present study follows both the precision of prenatal screening indicating a high degree of risk as established by confirmation or invalidation of the diagnosis by an invasive method of diagnosing genetic abnormalities, namely amniocentesis, and the consequences of this procedure.

The patient's name, age, last menstrual period, pregnancy age (first or last menstrual period or ultrasound), indication of amniocentesis, number of faces, possible fetal malformations diagnosed, Rh, and whether are known bearers of hepatitis B, C and HIV.

Patients were informed about the technique of performing this invasive diagnosis procedure, about its abortion risks, the possibility of obtaining "negative" cell cultures, the time needed to obtain the results (1-3 weeks), and the diagnostic alternatives.

It has been mentioned that amniocentesis can cause a feeling of abdominal pressure, abdominal cramps, amniotic fluid loss or vaginal bleeding after surgery, and that they will feel a pain while inserting the needle at the puncture site or feeling a pressure during fluid extraction amniotic. They have been informed that the procedure, although painful, can be performed with or without local anesthesia and that in local anesthesia it has only superficial effect and does not absolve the patient completely of pain during the procedure.

On the occasion of the check-up done before leaving the hospital, one-third of the patients were asked about the perceived pain or not during the procedure, and if so, if it was less pain than an 
intravenous puncture, less than it thought it would be or intense pain, stronger than an intravenous puncture and hard to bear.

All patients were advised to avoid excessive exercise and sexual intercourse on the day of amniocentesis and for the next 3-4 days, especially in patients with post-puncture abdominal colic and immediate presentation to a physician in case of complications such as fever, vaginal bleeding and amniotic fluid loss via vaginal route.

\section{RESULTS}

In the four analyzed years, a total of 6,097 pregnancies were recorded, of which 408 had an indication of an amniocentesis in order to establish a correct diagnosis. The dynamics of the number of tasks during the analyzed period show significant decreases compared to the first year, with $-14.65 \%$ in 2014 and $11.15 \%$ in 2015.

Of the total of 408 amniocentes in hospital, 114 were performed in 2012, 102 in 2013, 97 in 2014 and 95 in 2015. Comparing the amniocentesis dynamics with 2012, they have an even more pronounced decline, as follows: in 2013 their level decreased by $10.53 \%, 14.91 \%$ in 2014 , and $16.67 \%$ in 2015 due to the appearance of Harmony in maternal blood - a non-invasive prenatal screening test which allows for the risk assessment of trisomies 21,18 and 13 as well as the screening of sex chromosome abnormalities in the fetus..

In the four years in which the 408 amniocentesis was recorded, 251 were performed in patients up to 35 years of age, representing $61.5 \%$ and 157 being the number of patients over 35 years old with a percentage of $38.5 \%$. It was found that although the age over 35 years represents a risk factor for the development of genetic malformations, however, a higher percentage of all amniocentesis is the category of patients up to 35 years old, in Romania the amniocentesis protocol does not include compulsory amniocentesis after age 35 years, unlike other states.

The number of amniocentesis in week 15 is $14.22 \%$, in W16 - 34.56\%, in W17 - 26.72\%, W18 $-11.03 \%$, W19-7.35\%, W20 4.71\% and in W21 $1.96 \%$.

Although the largest number of amniocentesis was recorded in 2012 (114), only one case was recorded in which the amniocentesis indicated the maternal age, which is $0.88 \%$, in 2013 there is a slight growth, with a weight of $6.86 \%$, in 2014 $4.12 \%$, and in 2015 a decrease of $3.16 \%$. For the whole period, 15 indications of maternal age greater than or equal to 35 years were registered, the share in the total number of patients being $3.68 \%$.

It is noted that in 2012, out of the 114 amniocentesis, two had this method as an indication due to a chromosomal pathological history from which a patient has a Down syndrome family member in the family and another previously had Down syndrome, these indications representing $1.75 \%$ of amniocentesis indications for that year, there is no case with a chromosomal pathological history in 2013, another two patients with other indications were identified in 2014, one of the patients coming from the Down syndrome family, and the latter showing a pregnancy with a Turner syndrome. These two other indications represent $2.61 \%$, and in 2015 there is no case with a pathological chromosomal history.

Of the total of 408 patients who received amniocentesis, 149 were at the first pregnancy, ie $36.52 \%$, and 198 at the second pregnancy, ie $48.53 \%$, and 61 at the third or more much, ie $14.95 \%$.

The highest percentage is represented by patients with two previous abortions, namely 6.62\%, followed by those with three or more abortions, ie $5.88 \%$, and finally the patients who had a single abortion, $4.66 \%$.

A percentage of $23.28 \%$ comes from rural areas, and the remaining $76.72 \%$ from urban areas.

Despite the pregnancy, a fairly high percentage is smoking, namely $39.22 \%$, yet the majority of $60.78 \%$ is the group of non-smokers.

Half of the patients $47.79 \%$ developed rubella (German measles) in childhood, of which 77.95 were vaccinated and $31.28 \%$ were not vaccinated. Varicella in childhood occurred in $75.98 \%$ of patients.

The Down syndrome percentage to total pregnancies $(6,097)$ represents $1.6 \%$ one percent over European statistics, and Edwards syndrome $0.656 \%$. All patients whose children were diagnosed with Down syndrome or Edwards syndrome decided to discontinue the pregnancy.

Following the 408 amniocentesis, 4 abortions were recorded (two of them at 19 weeks gestation and three weeks after amniocentesis and two at 18 weeks, also 3 weeks after the amniocentesis).

The number of live births represents $96.56 \%$ of which $94.41 \%$ were born on term and $5.59 \%$ prematurely.

Maternal age in cases diagnosed with Down syndrome was $28,31,32,38$ and 40 years.

The 4 positive results for Edwards syndrome, along with 10 other positive Down syndrome findings, were due to the triplet screening test, the 
remaining 4 positive Down syndrome results were due to the positive Down syndrome score, and following the indication of maternal age 1 case resulted in Down.

By analyzing hierarchically the occurrence of chromosomal malformations, one can find that the Down syndrome is the first a frequency of 10 , then the occurrence being nuchal translucency.

\section{DISCUSSIONS}

Amniocentesis has become the prenatal diagnosis method most commonly used in pregnant women over 35 years of age or older and even younger women with a particular family or reproductive history at risk of hereditary congenital abnormalities.

In the study group, 157 pregnant women (38.48\%) requested prenatal diagnosis of Down syndrome aged 35 years or older and the association of pathological biochemical markers (triple, double test, AFP).

The frequency of structural chromosomal anomalies (translocations, deletions, duplicates, and inversions) did not show a similar relationship to numerical relationships in relation to advanced age.

The amniocentesis were all performed with real-time echographic probes, real-time ultrasound allowing visualization of the intrauterine content, so immediate changes in penetration depth or angulation of the puncture needle were made if necessary.

Pain is a multidimensional, complex sensation that varies in quality, intensity, duration of localization and discomfort from one individual to an- other. Intensity and pain disquiet is not directly related to the nature and extent of tissue damage (4).

Studies (5) have shown that women undergoing amniocentesis in the second trimester express concerns about the outcome of the production and potential fetal injury and loss of pregnancy.

The fear of pain is increasingly recognized as a factor contributing to an increased perception of pain and, implicitly, of the pain compartment (6). However, none of the patients questioned about the appearance of the pain experienced during the amniocentesis considered the perceived pain as being more large as an intravenous puncture, but did not mention before the proper procedure that they are afraid.

Of the 408 patients, 140 (34.31\%) required local anesthesia. There are studies in which local anesthesia is not used at all (7). Do not routinely use local anesthesia with $1 \%$ xylin in the practice of amniocentesis (8). Moreover, many studies in the literature, show that local anesthesia does not decrease maternal pain perception during amniocentesis $(9,10)$.

The respected technique during the entire anesthetic procedure with appropriate antiseptic solutions leads to avoiding or reducing the risk of infectious complications, amniotic, in some cases even very serious.

We have not experienced infectious complications in our series, which is consistent with many communications in our literature (11).

The incidence of abnormally colored amniotic fluid found on genetic pregnancy amniocentesis in the second trimester of pregnancy was reported in several series of literature as between 0.6 and $6.8 \%(12)$.

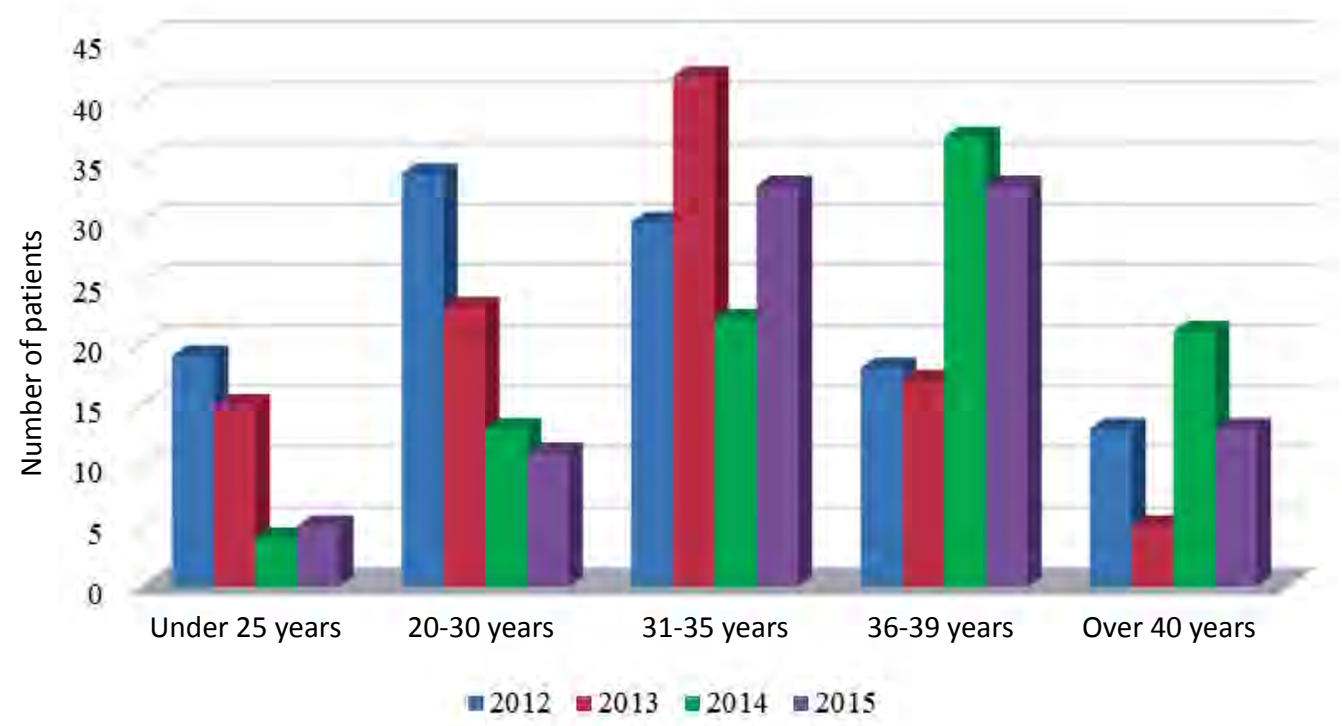

FIGURE 1. Distribution of patients on age groups 
The prognostic value of a dark amniotic fluid (large and green) on genetic amniocentesis is the increased risk of pregnancy loss (13). The incidence of spontaneous abortion in these cases is between $0 \%$ and $30 \%$.

The prevalence of chromosomal abnormalities in abnormally colored amniotic fluid is reported in different series between $0 \%$ and $4.8 \%$ and that of negative amniocyte cultures in these fluids is $0 \%$ to $20 \%$.

The prevalence of chromosomal abnormalities is considered to be higher in female genitalia than in monofetal pregnancies. We observed in our case an incidence of chromosomal abnormalities in accordance with the literature when analyzing the pathological karyotypes obtained in the 157 genetic amniocentesis performed for the advanced maternal age (over 35 years), where 155 were in case of monofetal pregnancies, and 2 in the case of gemorrhagic pregnancies.

We have not experienced infectious complications or bleeding or post-amniocentesis vaginal bleeding and only 2 cases of vaginal amniotic fluid loss.

Spontaneous abortion, a common complication of the technique, is the essential risk and has been observed in a single case less than 14 days after amniocentesis.

Vaginal loss of amniotic fluid immediately after amniocentesis is due to the fact that the amniotic fluid infiltrates the membrane and the wall of the uterine wall through the puncture site of the membranes, which then spreads through the cervix most often and spontaneously yields to approximately 48-72 hours after puncture.

In all cases where abnormal karyotype is known, pregnancy management (perinatal conduct) will be substantially affected, sometimes even avoiding unnecessary cesarean operation. Prenatal diagnosis of trisomy 21 allows parents to decide continuing or interrupting the affected gestation.

According to the literature, screening tests have an error margin in the diagnosis of fetal malformations, emphasizing the idea that amniocentesis can lead to a diagnosis of certainty,

Most specialists consider that amniocentesis is an invasive method, so use of this diagnostic method should be reduced. Therefore, amniocentesis is recommended as an invasive prenatal diagnosis when the risk of a detectable abnormality by this method is considered greater than the procedural risk of amniocentesis.

There are new tests that may reduce the practice of amniocentesis, but their costs are high and are not covered by the health care facility, which is why they are not accessible to any patient group.

Our study was on a broad population segment that had access to the screening tests (bitest, triple test), but not all of our patients had this possibility.

TABLE 1. The distribution of tests and cromosomial pathological antecedents

\begin{tabular}{|l|c|c|}
\hline Number of amniocentesis & $\mathbf{4 0 8}$ & $\mathbf{1 0 0 . 0 0 \%}$ \\
\hline Bitest & 131 & $32.10 \%$ \\
\hline Triple test & 258 & $63.23 \%$ \\
\hline Maternal age $\geq 35$ ani & 15 & $3.67 \%$ \\
\hline Down syndrome in family history & 2 & $0.49 \%$ \\
\hline Down syndrome pregnancy antecedents & 1 & $0.24 \%$ \\
\hline $\begin{array}{l}\text { Turner syndrome pregnancy } \\
\text { antecedents }\end{array}$ & 1 & $0.24 \%$ \\
\hline
\end{tabular}

Triple Load Test helps to detect neural tube defects and chromosomal anomalies such as trisomy 18 and 21. Thus, if a high level of alpha-fetoprotein is found in the blood, the fetus may have a neural tube defect (brain and spine), defects such as spina bifida or anencephaly. If, on the contrary, triple test results reveal a low level of alpha-fetoprotein (AFP) and estriol, this may be due to trisomy 21 (Down syndrome), trisomy 18 (Edwards syndrome), or other chromosomal abnormality.

All non-immunized Rh patients should be given anti-D immunoglobulin after amniocentesis.

Most of the surveyed patients reported that they thought that amniocentesis was a painful procedure, being comparable to literature data.

From the carried out research, according to the literature, it is important to warn the patient before amniocentesis that the pain perceived during the procedure is considered to be much less than expected.

Of the 408 cases studied, 14 pregnancies were discontinued because the parents decided they could not take responsibility for raising a child with malformations.

The final results of the 408 cases, about the post-amniocentesis pregnancy to pregnancy endpoint, claim that genetic amniocentesis presents a minimal risk of abortion or maternal infections, and fetal lesions are very rare.

\section{CONCLUSIONS}

In conclusion, we can say that we join the literature that shows that amniocentesis and other prenatal tests have managed to change the pregnancy from a worry-free period to a period of uncertainty and very important decisions (4 pregnant 
women with pathological karyotype have requested interruption of the pregnancy and the rest have remained under the burden of future fetal risks by the decision to continue the pregnancy, in addition to the pathological fetal karyotype).

Conflict of interest: none declared Financial support: none declared

\section{REFERENCES}

1. https://www.romedic.com/global-genetic (accessed June 25, 2019).

2. Prof. Dimitrie Nanu and co-autohors. Essential in Obstetrics, Ed. Medicală Amaltea 2015.

3. Copel JA, Pettker MC. Ultrasound Clinics of Amniocentesis: Technique and Complications. Contemporary OB/GYN, 2005.

4. McGrath PA. Psychological aspects of pain perception. Arch Oral Biol. 1994;39 Suppl:55S-62S.

5. Cederholm M, Axelsson O, Sjödén PO. Women's knowledge, concerns and psychological reactions before undergoing an invasive procedure for prenatal karyotyping. Ultrasound Obstet Gynecol. 1999 Oct;14(4):267-72.

6. McCracken LM, Faber SD, Janeck AS. Pain-related anxiety predicts non-specific physical complaints in persons with chronic pain. Behav Res Ther. 1998 Jun; 36(6):621-30.

7. Ferber A, Onyeije $\mathrm{Cl}$, Zelop CM, O'ReillyGreen C, Divon MY. Maternal pain and anxiety in genetic amniocentesis: Expectation versus reality. Ultrasound Obstet Gynecol. 2002 Jan;19(1):13-7.

8. Harris A, Monga M, Wicklund CA, Robbins-Furman PJ, Strecker MN, Doyle NM, Mastrobattista J. Clinical correlates of pain with amniocentesis. Am Obstet Gynecol. 2004 Aug;191(2):542-5.

9. Van Schoubroeck D, Verhaeghe J. Does local anesthezies at mid-trimester amniocentesis decrease pain experience? A randomized trial in 220 patients. Ultrasound Obstet Gynecol. 2000 Nov;16(6):536-8.
10. Gordon MC, Ventura-Braswell ADA, Higby K, Ward JA. Does local anesthesia decrease pain perception in woman undergoing amniocentesis? Am J Obstet Gynecol. 2007 Jan;196(1):55.e1-4.

11. Hess LW, Anderson RL, Golbus MS. Significance of opaque discolored amniotic fluid at second-trimester amniocentesis. Obstet Gynecol. 1986 Jan;67(1):44-6.

12. Taylor MB. Anderson RL, Golbus MS. 100 twin pregnancies in a prenatal diagnosis program. Am J Med Genet. 1984 Jul;18(3):419-22.

13. Zorn EM, Hanson FW, Greve LC, Phelps-Sandall B, Tennant FR. Analysis of the significance of discolored amniotic fluid detected at midtrimester amniocentesis. Am J Obstet Gynecol. 1986 Jun; 154(6):1234-40. 